\title{
System Engineering of Photonic Systems for Space Application
}

\author{
Michael D. Watson and Jonathan E. Pryor \\ NASA Marshall Space Flight Center, Huntsville, AL 35812
}

\begin{abstract}
The application of photonics in space systems requires tight integration with the spacecraft systems to ensure accurate operation. This requires some detailed and specific system engineering to properly incorporate the photonics into the spacecraft architecture and to guide the spacecraft architecture in supporting the photonics devices. Recent research in product focused, elegant system engineering has led to a system approach which provides a robust approach to this integration. Focusing on the mission application and the integration of the spacecraft system physics incorporation of the photonics can be efficiently and effectively accomplished. This requires a clear understanding of the driving physics properties of the photonics device to ensure proper integration with no unintended consequences. The driving physics considerations in terms of optical performance will be identified for their use in system integration.
\end{abstract}

Keywords: System Engineering, Optical Transfer Function, Optical Physics, Photonics, Image Jitter, Launch Vehicle, System Integration, Organizational Interaction

\section{INTRODUCTION TO SYSTEM ENGINEERING PRINCIPLES}

Engineering of photonics systems has many effects that must be accounted for in order to support the optical functions. The photonic system's interaction with the spacecraft and the environment has many different and complex effects. ${ }^{1,2}$ There are 4 system engineering aspects that must be understood in order to properly define and develop any system: Mission Context, Driving Physics, Organizational Dynamics, and Policy \& Law constraints. The Mission Context sets many of the environments and system parameters. Astronomy missions (x-ray, ultra violet, visible, infrared), solar physics, planetary observation, planetary landers, planetary rovers, or launch vehicle monitoring are all missions with very different environments and spacecraft busses. Knowing this context effects not only the environment but the type and tolerance of optics necessary to achieve the scientific or engineering objectives. Mission context also includes the launch vehicle and transport contexts. Some of the most significant constraints on the James Webb Space Telescope (JWST), for example, are defined by the available size of the launch vehicle fairings at the time of the mission definition. ${ }^{3}$ In addition to mission context, understanding the driving physics in for these systems is crucial to making decisions that support the mission goals and objectives. There are many physical effects which affect optical performance including mechanical stiffness and alignment, thermal transients, spacecraft stability and alignment, ${ }^{4}$ and contamination (e.g., out gassing, dust, micro meteorite, orbital debris). Defining the state variables for the spacecraft systems and their associated interaction effects provides a path to understand and properly manage the spacecraft and environmental effects on the photonics system performance. State variables define the state of each physical and information system and provide a means to efficiently manage the photonics within the spacecraft system and environment. These physics define the orbit, field of view, image stability, and even optics location. ${ }^{1}$ In launch vehicle monitoring applications, for example, aerodynamics is a driving constraint on location of cameras in flight. ${ }^{5}$ Organizational factors also play a major role in the definition of optical systems. The culture of the organization and the decision making processes within the organization greatly influence the efficiency, and potentially, the achievable solutions within the given budget and schedule constraints for the mission. ${ }^{6}$ These effects must be managed during the photonic system development, test, and integration with the spacecraft. This can be complicated when the photonics development organization must fit into the spacecraft organization culture and processes. Policy and Law provide many constraints on photonics systems, from environmental policies limiting materials and cleaning agents, to space policy limiting space debris 
generation and biological contamination of environments. Each of these factors play an important role in the definition, development, and integration of photonic systems into an overall spacecraft application.

\section{A. SYSTEM ENGINEERING PRINCIPLES: MISSION CONTEXT}

The mission context is the key factor in determining the photonic system environments. The type of mission sets spacecraft operational environments as well as the tolerances that various physical effects can have on the optical performance. Mission location of the photonic system is a primary consideration from the mission context. If the mission is conducted in planetary orbits (e.g., Hubble Space Telescope (HST) in Low Earth Orbit (LEO)) then effects such as atomic oxygen, MMOD, and space radiation must be managed or mitigated. ${ }^{7}$ In addition, protection of the photonics systems from solar intensity damage must be actively managed. Observatories located in interplanetary space (e.g., JWST) do not have these orbital environments while in operation and have lower levels of space radiation depending on the distance from the sun. ${ }^{8}$ Mission type is also a driving consideration in the system engineering of the optical system. Astronomy missions have extremely tight alignment and spacecraft stability requirements. Dim objects (i.e., at great distances) can require stable alignment for hours or days in order to obtain a high quality image. For these missions, mechanical vibration and thermal gradients must be managed or mitigated to allow for low aberrations. These missions also have to manage potential direct exposure to the sun during pointing maneuvers. Solar physics observatories (e.g., ) differ in that they are designed for direct viewing of the sun and actively manage the solar intensity exposure and effects on the optics. Planetary observers (e.g., TRIMM) must deal with the planetary orbital environments. Orbital altitude is an important factor in the type of environment and stability of the spacecraft observation platform. ${ }^{2}$ Planetary landers and rovers (e.g., MSL) must deal with the planetary atmosphere (corrosive or benign, etc.) and the surface dust. Temperature extremes on the surface must also be managed by the system. Launch Vehicle photonics (e.g., SLS) must deal with the launch site environment (generally in tropical locations having high humidity and temperatures) as well as the aerodynamic and acceleration forces and vehicle vibration during ascent flight. In addition to setting the environment for system operations, the mission type also establishes the type of optics required including x-ray optics, normal incidence reflective and/or refractive optics, coronagraphs, etc. Finally, mission context defines the transport of the optical system from the Earth's surface to the mission environment. This includes limitations from launch vehicle environments (fairing length and diameter, thermal environments, shock and vibration, and acceleration forces) and in-space transport systems (e.g., transport stages or upper stages for Earth Departure and solar or planetary orbit insertion). The optics must tolerate these environments while stowed or operate in these environments depending on the context.

\section{B. SYSTEM ENGINEERING PRINCIPLES: DRIVING OPTICAL PHYSICS}

Once mission context has defined the operating environment and type of optical system for the mission, the physical effects and information logic will fully define the system. The Optical Transfer Function, using Fourier optics relationships from the object to the image, provides the physical equations to define the optical system. ${ }^{9,10}$ The OTF is unique to each specific optical system (lenses, mirrors, apertures, image planes, shutters). In addition, tolerances for thermal gradients, image stability, platform vibrations, aberrations, and intensity are all definable within the OTF by allowing variation of integration limits of the pupil functions, transfer distances, and intensity factors (for intensity effects). Writing the OTF as the spatial transfer function takes the general form as,

$$
\text { 1) } \iint_{-\infty}^{\infty} \psi_{o b j} s_{f} d x d y
$$

Where the spatial filter, $\mathrm{s}_{\mathrm{f}}$, represents the system aperture, lens, and mirror geometries and optical element spacing in the $\mathrm{x}, \mathrm{y}$, and $\mathrm{z}$ dimensions. ${ }^{11}$ In incorporating this with the spacecraft system, the spatial dimensions of the optical system can vary due to thermal gradients, vibrations, and mechanical misalignment. In addition, sf captures the 
system variation due to contaminations on the optical elements from dust or outgassing. These contamination effects can be rather complicated leading to both intensity effects and image aberration effects.

The OTF can be very complex and the system engineer ensures that all aspects of the system due to physical effects from the environment and spacecraft are properly represented and the associated tolerances are met. The inter relationship between the spacecraft systems, environments, and optical system can be intricate. The aperture or pupil functions, $\mathrm{p}_{\mathrm{f}}$, as a part of the spatial filter vary as functions of thermal expansion $\left(\alpha_{\mathrm{v}}\right)$, heat transfer rate $(\dot{\mathrm{Q}})$, temporal vibration $(d(t))$, and shock $\left(a_{\text {shock }}\right)$. Thus, $p f=f\left(\lambda, \alpha_{v}, \dot{Q}, d(t), a_{\text {shock }}\right)$ These must be understood to ensure all system perturbances can be controlled by the spacecraft or tolerated by the optical system. The system state variables are key representations of optical parameters and physical tolerances of the optical system. By defining the state variables with clear relationships between the spacecraft physics and the optical system, a well-structured system can be defined which accounts for all spacecraft and environmental effects on the optical system.

In addition, the image must be digitized for downlink and information on the optical system must be collected for subsequent scientific and engineering analysis. This information provides a representation of the physical with the addition of measurement uncertainties, gibbs phenomena (ringing due to higher frequency data truncation), and other affects that result in deviations of the information states from the actual physical state of the optical system.

\section{SYSTEM ENGINEERING PRINCIPLES: ORGANIZATIONAL STRUCTURE AND RELATIONSHIPS}

In considering organizational structure and relationships, it is important to establish that the optical engineer is responsible for the optical design, not the system engineer. The system engineer focuses on the effects and relationships between the optical system, spacecraft systems, and environments. Managing these relationships is the key to smooth optical system integration and a successful mission function.

The organizational challenge for the optical design team is the integration with the larger spacecraft design and operations organization. These teams can have very different approaches and may not always appreciate the extreme (on the order of wavelength) tolerances that optical systems require. The decision making processes used within the spacecraft organization may be different from the optical design organization. The system engineer helps ensure that these organizations understand each other and that information flows together smoothly. An important relationship to establish early is the justification for flight readiness. For observatory type missions, this can be strongly driven by the optical design and the effects of spacecraft system on the optical system. On the other hand, in application were optical systems are secondary objectives, or where they are in system support functions (i.e., launch vehicles), the optical design organization will need to provide information and support the spacecraft or launch vehicle team in certifying the full system is ready for flight.

The Organizational structure and relationships define the efficiency which an organization can accomplish a specific optical system development. It is not a question of simplicity or complexity but rather an establishment of an organizational structure to support the specific optical system development. Ensuring that the organizations work smoothly together greatly facilitates and efficient and successful system development.

\section{SYSTEM ENGINEERING PRINCIPLES: POLICY AND LAW CONSTRAINTS}

Policy and law provide guidance and constraints on many potential solutions for a particular optical system design. Environmental laws and policies restrict certain chemicals used in optical materials and in optic cleaners that may limit design solutions. Various international treaties are in place which control space debris and the responsible disposal of Earth orbiting assets. These treaties form constraints on orbits, and possibly, mission life in order to minimize debris in Earth Orbit. Occupational health and safety regulations also place constraints on manufacturing, 
assembly, and test operations to ensure the workforce is properly protected. These can lead to increased cost or schedule if not planned for in the development schedule and optical system design. The applicable policies and laws can effect each optical system differently and the system engineer should ensure these are understood and addressed as the system is developed and operated.

\section{OPTICAL SYSTEMS ENGINEERING EXAMPLES}

Having established the system engineering framework within which optical systems can be effectively integration into space missions, several examples can be investigated to see these effects. To illustrate these principles, the integration of an imaging system onto a launch vehicle is briefly considered.

\section{A. SYSTEM ENGINEERING OF LAUNCH VEHICLE IMAGING SYSTEMS}

For an imagery system on a launch vehicle, the mission is to provide video data for analysis of debris anomalies, physical malfunctions, and separation tracking events for multi-stage rockets. The imagery system on a launch vehicle is a complement to the main mission of successfully delivering a payload into low-Earth orbit and beyond, and provides invaluable information if something goes awry during a lunch event. The imagery system must not interfere with the main launch objective, and it must deliver data to the ground in the event that an anomaly occurs that is only accessible and understandable via imagery. Figure 1 illustrated the Space Launch System launch vehicle.

Launch vehicle imaging systems physics are driven by the launch vehicle environments affecting spatial distances and image stability. These imaging systems primarily consist of a set of cameras, lenses, and recorders combined with launch vehicle specific housings and harnesses. These systems must work within limited downlink capabilities shared with other systems to stream video data to the ground for storage and distribution. If the launch vehicle, stages, or boosters are recovered after flight, imagery data may be recovered post-flight. In the case of the Space Launch System (SLS), video data will only be streamed to the ground and stored for later analysis.

Although video quality of recovered data is higher than streamed data since larger image file sizes can be stored than can be streamed, trade-offs must be made at the system level to determine what is viable given image resolution, bandwidth, budget, and schedule constraints.

Cameras are initially selected based on a combination of image quality, sensor type,

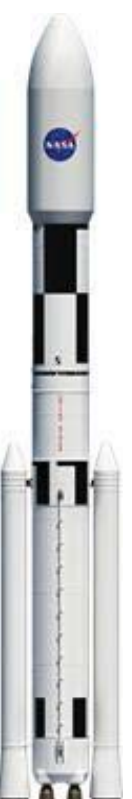
output signal type, and low-light capabilities. These cameras typically are not consumer level cameras (with externally accessible controls), but designed with engineering tasks in mind (remote computer controlled settings). The number of cameras, recorders, and locations of each are selected based on a combination of different spatial reference factors. First, the desired areas of observation are selected, and from this, the number of desired cameras and their locations. Lenses can be selected based on the viewing angle, camera field of view, and required image size and resolution (magnification and aberrations) once their location (spatial distance from desired object plane) has been determined. The use of Computer Aided Design (CAD) software is often used for image simulation when deciding where and at what angles to place the cameras. The CAD system provides a useful tool to analyze the spatial distances between the object plane and camera lenses. This also aids in lens selection as tradeoffs are made with magnification and image quality. The recorders are selected based on video encoding capabilities and the quality of the compressed video. High quality image compression is typically required on a launch vehicle due to limited bandwidth. This bandwidth is shared with many other subsystems making this a system constraint on the image storage and transmission capabilities. The allotted video bit rate will drive the recorder selected based on its ability to compress the video at a high quality. The location of the cameras and recorders on the launch vehicle are system integration considerations and do not drive the optical parameters of the 
system. These system integration considerations have major design implications for the cameras' housings and cabling linking them together as the photonics system is integrated with the other launch vehicle systems.

Image jitter, related to the vibration frequencies of the launch vehicle during flight, can present a problem to the imaging system. ${ }^{4}$ Jitter is a spatial variation with time (image motion). The object plane and/or image are moving spatially with respect to each other based on the frequency of the vibration and the spatial separation between the object and image planes. This is typically a sinusoidal variation and results in a zeroth order Bessel function, $\mathrm{J}_{0}(\pi, \mathrm{kx}, \mathrm{ky}),{ }^{12}$ in the optical transfer function. Understanding the mission context and the physics of the source and transmission of the vibration frequencies, there are camera selection decisions that can be made to reduce (but typically not eliminate) the image jitter. ${ }^{13,14,15}$ The imaging system mission does not require high resolution, rather the image must be clear enough to identify and track the motion over a few frames while in the camera field of view. The key in this case is to address the time varying aspect of the vibrational wave to minimize the spatial variations that manifest as jitter. ${ }^{16}$ Selecting a camera with a large sensor can aid in the reduction of image jitter by allowing more light to be collected on the sensor in the same timespan as a smaller sensor. This allows for a shorter exposure time which minimizes the spatial variations while preserving image quality. The selection of the exposure time is typically the fastest available for the image detector to minimize the spatial motion that occurs due to jitter, but this must be weighed against acceptable image noise and the depth of field that needs to be imaged. The necessary depth of field will likely help select the aperture setting which will help select the ISO setting and exposure time. On a launch vehicle, choosing a shorter exposure setting at the cost of a higher ISO setting is worth a more noisy image for less image blur. Other factors such as ISO performance, resolution, and shutter read-out type also play a factor in camera selection for its low-light performance, image detail, and global shutter, respectively.

There are other mechanical factors that can help to reduce image jitter. Removing moving parts from a lens, and having a lens assembly without focusing controls and aperture selection help to reduce parts of the camera subject to vibration induce motion. By reducing these moving components and adding a vibration reduction film between the lens and camera, image jitter can be noticeably reduced. Also, by creating a camera housing that secures both the lens and the camera separately, a more robust camera system is created adding to the the spatial stability of the system with a reduction in image jitter.

The housings for cameras and recorders are driven by operating environment considerations and integration considerations. They are designed to protect the cameras and recorders from the environment. The must provide adequate protection from thermal, vibration, and shock environments which protect both the optical image quality and physical damage to the imaging system. Engineering tests influence design decisions made for the housings. These environmental tests typically lead to customizations to be made to the cameras and lenses in order for the system to properly operate in the launch vehicle environment. In the case of SLS, customizations were made to ruggedize the cameras and lenses. The housings also harbor electrical circuits that provide voltage conversion, power filtering, and microcontroller circuits. These microcontrollers contain control software for powering on and off different cameras and recorders and controlling the streaming of imagery data to the ground based on the flight time-line and bandwidth. It may also control lighting systems designed to illuminate internal scenes based on separation events.

The cable harness will be designed to withstand the launch environments, but also to prevent video signal degradation and reduce power loss while still meeting mass requirements. The type of video cables drives connector selection, cable selection, and cable routing. These selections have huge implications on schedule due to delivery time estimates of different connectors and cabling. Some connectors/cables will have lead times exceeding 12 weeks, while video recorders, cameras, and lenses can exceed 20 weeks lead time.

The organizational relationships are important during the manufacturing phase. The manufacturing process can include the design and production of housings, electronic board design and assembly, and cable harness design and assembly. The engineering design team must work closely with the manufacturing team to ensure proper 
component selection based on heritage projects. The synergy between engineering and manufacturing plays a huge role in the timely production of the final imagery system that is installed on the launch vehicle. Proper understanding of the needs of the manufacturing group to understand the optical requirements and how to state these in an understandable manner are a key to successful product implementation. In addition, policies of the organization also play a major role on schedule and hardware selection.

Organizational interactions are the sources of many unintended consequences where the importance of subtle mechanical factors can have a large effect on optical performance. Launch vehicle vibrations generally affect the strength of materials and the material fracture mechanics which determines the components ability to survive the launch environments. These are important considerations for optical systems as well, but the vibrations induced image jitter is a large optical performance impact which often needs additional attention in the mechanical design, manufacturing, and assembly. While launch vehicle imaging systems are less sensitive to material selection from a strength of material and fracture mechanics view point (many materials satisfy these constraints), the material selection process must take into account the material off-gassing possibilities for the pressure and thermal environments experience during ascent flight. If the lens was to be coated with a materialthat can off-gas in these environments, image quality is reduced and the image could be lost. This is a factor not generally a concern for other mechanical systems on a launch vehicle and must be carefully explained and reviewed with the mechanical design team. Other design considerations, such as the lens staking made previously, would only be discovered to be effective in the engineering imagery lab where these types of considerations are being made. Housing window selections are another area that require imagery data considerations rather than pure mechanical strength considerations. From a mechanical engineering standpoint, some materials may be desired due to strength and rigidity, while from an imaging standpoint, optical clarity may be a more important factor. It is important for the imaging, mechanical design, and manufacturing teams to communicate these issues and come to a solution that satisfies the imaging requirements, mechanical and thermal environments, and manufacturing and assembly processes. .

Requirements placed on the manufacturing process by the organization may have huge schedule impacts and limit the selection of hardware components. Systems that are in place for drawing release and limitations on how hardware is purchased and inspected can be limiting factors from a scheduling perspective. When a launch date is selected for the vehicle, and schedule limitations are imposed by organizational requirements, this may in turn drive hardware selection and engineering designs and testing due to these time limitations.

Designing an imagery system for a launch vehicle such as SLS requires a different set of priorities when compared to astronomy missions. While astronomy missions include the optics as a primary payload, the imaging system on a launch vehicle compliments the vehicle by providing imagery data that can be analyzed at a later date. The primary mission of SLS is to carry a payload safely to low-Earth Orbit and beyond. Its imagery system is there to provide video data for analysis of debris anomalies, physical malfunctions, and separation tracking events. The launch vehicle provides a challenging environment for an imaging system where bandwidth and schedule limitations require the imaging team to think and design creatively.

The imaging system as a whole is comprised of trade-offs between schedule, manufacturing, engineering, testing, processes, hardware selection, and system level imaging requirements. It is a complex system that requires the cooperation of many different groups across an organization.

\section{SUMMARY}

System engineering of photonic and optical systems is a complex functions. The mission context varies greatly among optical application mission types from observatory missions to planetary missions to launch vehicle applications. The mission location defines the operational environments (i.e., Earth orbit, interplanetary space, atmospheric operation, planetary surface operation) of the optical system. The method of transport from Earth to the 
final location also drives the optical design depending on the mission type. The physics of the optical system define the integration aspects with the spacecraft systems and environments. The Optical Transfer Function (OTF) describes the optical system and provides a basis for the level of tolerances for optical system integration with the spacecraft. The spatial filter is driven by spacecraft and environmental interactions and is a function of thermal expansion $(\square \mathrm{V})$, heat transfer rate $(\dot{\mathrm{Q}})$, temporal vibration $(\mathrm{d}(\mathrm{t})$ ), and shock (ashock). The system engineer is responsible for the integration of the optical system with the spacecraft and must understand and appreciate the complex interactions these functions represent and the level of tolerances required by the optical system. The optical engineer is responsible for the optical system design coordinating with the system engineer to ensure proper system integration. Organizational interactions are important aspects to manage, especially when the optical design team must interact with a spacecraft organization that operates and functions differently. Determining how the optical system drives or supports the determination of flight readiness of the mission is an essential system engineering function. Policy and law provide constraints on the design solution and operation of the spacecraft in space. These policies and laws, including international treaties, must be identified early and accommodated in the optical system design as applicable. System engineering plays an essential role in properly integrating optical systems into the spacecraft to achieve mission success.

\section{REFERENCES}

${ }^{1}$ Bos, B. J., Davila, P.S., Jurotich, M., Hobbs, G, Lightsey, P., Contreras, J and Whitman, T., "The James Webb Space Telescope instrument suite layout: optical system engineering considerations for a large, deployable space telescope", Optical, Infrared, and Millimeter Space Telescopes, edited by John C. Mather, Proceedings of SPIE Vol. 5487, 2004.

${ }^{2}$ Thompson, P. L., Hill, P. C., "Conceptual optical design and system engineering of the CLARREO / RS (Reflected Solar) instrument suite", Imaging Spectrometry XVII, edited by Sylvia S. Shen, Paul E. Lewis, Proc. of SPIE Vol. 8515, 2012

${ }^{3}$ Feinberg, L., Cohen, L., Dean, B., Hayden. W., Howard, J., Keski-Kuha, R., "Space Telescope Design Considerations", Optical Engineering, 51(1), 011006, January 2012.

${ }^{4}$ Brady, D. H., Aaron, K., Stumm, B., Bronowicki, J. A., Chan, I., S., and Morris, P., A., "Structural design challenges for a Shuttle-launched Space Interferometry Mission", Interferometry in Space, Michael Shao, Editor, Proceedings of SPIE Vol. 4852, 2003.

${ }^{5}$ Dong, J., Xia, Y., Yu, Q., Su, A., and Hou, W., "Instantaneous video stabilization for unmanned aerial vehicles", Journal of Electronic Imaging 23(1), 013002, Jan-Feb 2014.

${ }^{6}$ Wyman, C., L., "Optical Systems Engineering: A Tutorial”, SPIE Vol. 187, System Aspects of Electro- Optics, 1979.

'Watson, M.D., Minow, J., Altstatt, R., Wertz, G, Semmel, C., Edwards, D., and Ashley, P. R.,, "Space application requirements for organic avionics", Photonics for Space Environments IX, edited by Edward W. Taylor, Proceedings of SPIE Vol. 5554, pp. $92-105,2004$.

${ }^{8}$ Watson, M. D., "Photonics on the Mission to Mars", Proc. SPIE 8876, Nanophotonics and Macrophotonics for Space Environments VII, September 24, 2013.

${ }^{9}$ Scott, R., M., "Optical Engineering", APPLIED OPTICS, Vol. 1, No. 4, July 1962, pp. 387-397.

${ }^{10}$ Dow, F., D., "Optical Image Evaluation and the Transfer Function", APPLIED OPTICS, Vol. 2, No. 4, April 1963, pp. 335-350.

${ }^{11}$ Banerjee, P. P., Poon, T., “Principles of Applied Optics", Irwin Publishing, 1991.

${ }^{12}$ Brown, Earle, "Modern Optics", Kreiger Publishing, NY, 1974, pp. 498-9.

${ }^{13}$ Lee, D., Yoon, J., Han, J., " Integrated Framework for Jitter Analysis Combining Disturbance, Structure, Vibration Isolator and Optical Model", Active and Passive Smart Structures and Integrated Systems 2012, edited by Henry A. Sodano, Proc. of SPIE Vol. 8341, 2012.

${ }^{14}$ Genberg, G. L., Michels, G. J., Doyle, K. B., "Integrated Modeling of Jitter MTF due to random loads", Optical Modeling and Performance Predictions V, edited by Mark A. Kahan, Proc. of SPIE Vol. 8127, 2011. 
${ }^{15}$ Gibson, S., Tsao, T., Herrick, D., Beairsto, C., Grimes, R., Harper, T., Radtke, J., Roybal, B., Spray, J., Squires, S., Tellez, D., Thurston, M., "Adaptive Jitter Control for Tracker Line of Sight Stabilization", Advanced Wavefront Control: Methods, Devices, and Applications VIII, edited by David C. Dayton,

Troy A. Rhoadarmer, Darryl J. Sanchez, Proc. of SPIE Vol. 7816, 2010.

${ }^{16}$ Jian-ting,, L., Zhi-zhong, W., Xin, G., Qiang-lin, X., Yu-jun, X., “Control techniques of delay-time jitter from the echo-transponder satellite laser ranging", Optoelectronic Imaging and Multimedia Technology II, edited by Tsutomu Shimura, Guangyu Xu, Linmi Tao, Jesse Zheng, Proc. of SPIE Vol. 8558, 2012. 